\title{
Steffi Schadow
}

\section{Kants Begriff moralischer Verpflichtung}

Was heißt es nach Kant, dass wir moralisch verpflichtet sind? Und worin gründet moralische Verpflichtung? Ich werde im Folgenden zunächst Kants Verwendung der Begriffe von Pflicht und Verpflichtung untersuchen. Sodann wende ich mich der Problematik der Moralbegründung zu und erörtere die ,Faktumsthese' aus der Kritik der praktischen Vernunft. Die Funktion der ,Tat der Vernunft', die jede Art von Sollen erst konstituiert, erschließt sich, so meine These, im Rückgriff auf in der Kritik der reinen Vernunft eingeführte handlungstheoretische Voraussetzungen. Wenn wir die Vernunft als Vermögen der Freiheit verstehen, können wir Verpflichtung und Sollen als reine Vernunftbegriffe denken, die durch diese Vernunft selbst konstituiert sind. ${ }^{1}$

\section{Kants Begriff moralischer Verpflichtung}

Kants Begriff moralischer Verpflichtung hat handlungstheoretische Grundlagen. Den Begriff der Handlung gebraucht Kant zunächst für alles, was ein Bewirken ist: das, „wodurch etwas geschieht“2. Menschen haben aufgrund ihrer vernünftigen Veranlagung „Kausalität durch Freiheit“"3, d.h. sie können aufgrund von Vor-

1 Zur historisch-systematischen Einordnung des kantischen Verpflichtungsbegriffs siehe Klemme, Heiner F.: Freiheit, Recht und Selbsterhaltung. Zur philosophischen Bedeutung von Kants Begriff der Verbindlichkeit. In: Normativität des Lebens - Normativität der Vernunft? Hg. von Markus Rothhaar und Martin Hähnel. Berlin/Boston 2015, 95-116. Kants Verpflichtungsbegriff in den Ethik-Vorlesungen beleuchtet Sensen, Oliver: Moral Obligation and Free Will. In: Kant's Lectures on Ethics. A Critical Guide. Ed. Lara Denis and Oliver Sensen. Cambridge 2015, 138-155. Zum Zusammenhang von „Freiheit“ und „Verpflichtung“ in GMS III siehe z. B. Quarfood, Marcel: Kant’s Practical Deduction of Moral Obligation in Groundwork III. In: Kant und die Berliner Aufklärung. Akten des IX. Internationalen Kant-Kongresses. Hg. v. Volker Gerhardt u. a. Berlin 2001, 72-79 sowie Schönecker, Dieter (Hg.): Kants Begründung von Freiheit und Moral in ,Grundlegung' III. Münster 2015. Der Weg der Analyse von Kants Verpflichtungsbegriff über die Faktumsthese ist m. E. noch wenig beschritten worden. Für ein Beispiel siehe Lueck, Bryan: Kant's Fact of Reason as Source of Normativity. In: Inquiry, 52/6 (2009), 596-608.

2 Kant: KrV A544/B572. Vgl. auch Kant: KrV B431f.; Kant: KrV A618/B646. Zu Kants Handlungsbegriff vgl. Schadow, Steffi: Handlung. In: Kant-Lexikon in 3 Bänden. Hg. v. Marcus Willaschek, Jürgen Stolzenberg, Georg Mohr und Stefano Bacin. Bd. 2. Berlin/Boston 2015, 992-995.

3 Kant: KrV: A538/B566.

Steffi Schadow, [Adresse]

https://doi.org/10.1515/9783110467888-050 
stellungen und Vermögen Ursache von Wirkungen in der empirischen Welt sein. Da Kausalität nach Kant immer gesetzmäßig verfasst ist ${ }^{4}$, muss auch die Kausalität aus Freiheit auf ein Gesetz bezogen sein. ${ }^{5}$ Dieses Gesetz der Freiheit ist das ,moralische Gesetz ${ }^{6}{ }^{6}$ Wenn Menschen handeln, dann bestimmen sie ihren Willen selbst durch das Gesetz der Freiheit im „Reich[.] der Zwecke“.7

„Pflicht“ fällt für Kant zunächst unter den allgemeinen Begriff der praktischen Notwendigkeit, denn sie betrifft eine Handlung, die zu tun ist. Gemäß der schon vorkritischen Unterscheidung von necessitas problematica und necessitas legalis unterscheidet Kant dabei zwischen verschiedenen Arten der Notwendigkeit von Handlungen: Eine Handlung kann als Mittel zur Verwirklichung eines Zwecks geboten sein oder aber weil sie auf einen Zweck bezogen ist, der an sich selbst verwirklicht werden soll. ${ }^{8}$ Im letzteren Fall gebietet keine pragmatische Regel, sondern das moralische Gesetz als Gesetz der Freiheit. Die Handlung ist daher nicht nur notwendig in Bezug auf einen durch sie realisierbaren Zweck, sondern sie wird durch das Gesetz mit absoluter Notwendigkeit und ausnahmslos geboten. ${ }^{9}$ Aufgrund dieser unbedingten Nötigung ist die Handlung für mich verbindlich. ${ }^{10}$ Dass ich die Handlung, zu der ich verbunden bin, tun muss, bedeutet daher weder, dass ich durch das Verfolgen kontingenter Zwecke auf diese Handlung festlegt bin, noch dass mein Handeln schlichtweg determiniert wäre wie rein natürliches Geschehen. Das Müssen einer für mich verbindlichen Handlung entspringt im Gegenteil aus der Tatsache, dass ich zu ihr genötigt werden kann, weil es ein moralisches Gesetz gibt und die Handlung in meiner Gewalt steht. ${ }^{11}$

Was kennzeichnet nun genauer die Pflicht und die moralische Nötigung, durch die eine Handlung für mich verbindlich ist? Im Nachlass zur Metaphysik der Sitten findet sich folgende Bemerkung: „Ein vernünftiges Wesen hat Pflichten wenn die Freyheit seiner Willkühr durch ein Gesetz eingeschränkt wird [...]. " ${ }^{12}$ Wie

4 Vgl. Kant: GMS, AA 04: 412.26, $446.15 \mathrm{f}$.

5 Vgl. z.B. Kant: GMS, AA 04: 446.15-21.

6 Vgl. Kant: GMS, AA 04: 447.05f. und Kant: KpV, AA 05: $47.30 \mathrm{f}$.

7 Kant: GMS, AA 04: 438.20. Zur fundamentalen Bedeutung der Fähigkeit der eigenen Zwecksetzung für die Ethik vgl. Kant: TL, AA 06: 381.18-34.

8 Vgl. Kant: UD, AA 02: 298.10-16.

9 Zur Unbedingtheit moralischer Vorschriften in Form ihrer absoluten Notwendigkeit vgl. Kant: GMS, AA 04: 389.11-13; 416.20-22, in Form ihrer Allgemeingültigkeit (,universalitas“) im Gegensatz zu „bloße[r] Gemeingültigkeit (generalitas)“ vgl. Kant: GMS, AA 04: 424.30f.

10 Vgl. u. a. V-Mo/Mron II, AA 29: 612.03-05; V-MS/Vigil, AA 27: 578.08-10; Kant: MS, AA 06: 223.01-03.

11 Vgl.V-Mo/Kaehler(Stark): 36.34-36-37.01-03. Zum Unterschied zwischen „Nothwendigkeit“ und „Nötigung“ vgl. V-Mo/Mron II, AA 29: 611.06.

12 Kant: VARL, AA 23: 344.19f. 
Kant hier und andernorts ausführt, stimmen die Handlungen der freien Willkür eines endlichen Vernunftwesens aufgrund seiner Affizierbarkeit durch Neigungen und Bedürfnisse nur kontingenterweise mit dem Moralgesetz überein. ${ }^{13}$ Die objektiv notwendige Handlung ist hier, wie Kant es ausdrückt, nicht zugleich subjektiv notwendig. ${ }^{14}$ Das Gesetz wird daher von einem Wesen mit einem unvollkommenen Willen im Modus des Imperativs erfahren. ${ }^{15}$ Da der kategorische Imperativ als „Formel des Gebots“16 Handlungen nach verallgemeinerbaren Maximen gebietet und als solches ein absolutes Gebot für alle Moralsubjekte ist, steht er für das, „was Verbindlichkeit sei“"17. Verbindlichkeit ist daher ,nur“ durch absolute Nötigung in kategorischen Imperativen gegeben. ${ }^{18}$

Die „moralische Nöthigung“, die durch diese Konstellation entsteht, bezeichnet Kant als Verpflichtung. ${ }^{19}$ Das moralische Gesetz, auf das sich die Pflicht dem Inhalt nach bezieht, ist ein Gesetz „für alle vernünftige[n] Wesen, so fern sie überhaupt einen Willen [...] haben“20. Aber es nötigt und $z$ wingt $^{21}$ im Sinne von: bindet nur den Willen eines endlichen Vernunftwesens. ${ }^{22}$ Dabei ist es die Struktur des Wollens, die den Doppelcharakter der Nötigung erschließt: So ist die menschliche Willkür zum einen eine sinnliche Willkür, weil sie für sinnliche Impulse empfänglich ist. Sie ist aber trotz dieser Affizierbarkeit eine freie Willkür, nämlich das Vermögen, sich von sinnlichen Einflüssen nicht ,nezessitieren` zu lassen. Die Nötigung durch das Gesetz ist dementsprechend eine Funktion der Beziehung des Gesetzes auf Adressaten, deren Willkür zugleich affizierbar und frei ist. Der Grund der Verpflichtung liegt nach Kant daher in der Einschränkung der Freiheit der Willkür durch ein Gesetz und nicht schon in der bloßen Existenz des Gesetzes.

Dass ich verpflichtet bin, heißt demnach, dass ich einem Moralgesetz unterstehe, das mich objektiv nötigt. In der Pflicht ist die Forderung enthalten, mich dem objektiven Gehalt des Gesetzes entsprechend subjektiv zu nötigen. Wo ein praktisches Gesetz ist, da ist für ein Wesen mit einem unvollkommenen Willen die

13 Vgl. z.B. Kant: KpV, AA 05: 32.25-31.

14 S. z. B. V-Mo/Kaehler(Stark): 29.27-33.

15 Vgl. z.B. Kant: KpV, AA 05: 32.21-23.

16 Kant: GMS, AA 04: $413.10 \mathrm{f}$.

17 Kant: MS, AA 06: 225.06f.

18 Vgl. Kant: MS, AA 06: 222.03f.

19 Vgl. Kant: TL, AA 06: 487.17f; V-Mo/Mron II, AA 29: 612.06f.

20 Kant: KpV, AA 05: 32.10f.

21 „Alle Obligation ist eine Art von Zwang“ (V-Mo/Collins, AA 27: 269.19). Zum Zusammenhang von Nötigung und Zwang vgl. V-Mo/Kaehler(Stark): 45.

22 Vgl. z.B. Kant: KpV, AA 05: 32.21-23. 
Verpflichtung, diesem Gesetz Folge zu leisten. Verpflichtung bzw. Verbindlichkeit bezeichnet daher den Sachverhalt, dass es ein Gesetz gibt, das nötigt und durch das ich mich selbst als verbunden betrachte, ${ }^{23}$ während Pflicht die Handlung ist, die zu tun man verbunden ist. ${ }^{24}$

Wie aber entsteht die Nötigung und worauf geht sie zurück? Nach Kant gehört es zur Bedingung von Verpflichtung überhaupt, dass ich mir meiner Pflicht prinzipiell bewusst werden kann. Der Pflichtbegriff ist deshalb folgendermaßen zu spezifizieren: Weil es ein Gesetz gibt, dessen Forderung mir prinzipiell bewusst ist, gibt es überhaupt eine Pflicht. Verpflichtet bin ich insoweit, als ich die Geltung eines (objektiv gültigen) moralischen Gesetzes (subjektiv) erkenne oder erkennen kann. Kant hat diesem Gedanken an prominenter Stelle in der Grundlegung Ausdruck verliehen: „Pflicht ist die Nothwendigkeit einer Handlung aus Achtung fürs

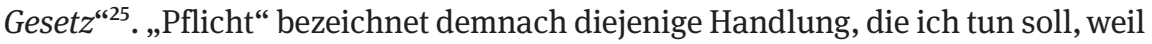
ich mir eines Gesetzes bewusst bin, das dies gebietet. Gemäß Kants Definition der Achtung als „Bewußtsein der Unterordnung meines Willens unter einem Geset$z^{\text {“ } 26}$ hebt die Pflichtdefinition an dieser Stelle primär den kognitiven Aspekt der Achtung hervor und versteht sie als eine Aufmerksamkeit dafür, dass es ein Gesetz gibt, das den Willen bindet. ${ }^{27}$

Dafür, dass es möglich ist, dass das Gesetz auf den Willen wirkt, liefert nun die „Vernunft allein die objectiven Gründe“28. Das heißt: Da Menschen über Vernunft verfügen, sind sie für objektive Gründe im Allgemeinen und für einen unbedingten objektiven Grund wie das moralische Gesetz im Besonderen empfänglich. Das sittliche Bewusstsein, das durch keine anderen ,Einflüsse“ vermittelt ist, bezeichnet Kant als Achtung. Sie ist daher selbst eine Form des Wissens, das sich auf ein rein formales Konzept, das moralische Gesetz, bezieht. Zugleich ist die Achtung als moralisches Gefühl eine praktische Einsicht, durch die Kant verdeutlicht, dass Menschen aufgrund ihrer Vernunft moralisch sensibel und für rationale Gründe auch praktisch empfänglich sind, so dass sie sich von ihnen in ihren Handlungen leiten lassen. ${ }^{29}$

23 Vgl. Kant: TL, AA 06: 417.27.

24 Vgl. Kant: GMS, AA 04: 439.33f; Kant: MS, AA 06: 222.31.

25 Kant: GMS, AA 04: 400.18f.

26 Kant: GMS, AA 04: 401.23f.

$27 \mathrm{Zu}$ den verschiedenen konzeptionellen Aspekten der „Achtung“ siehe Schadow, Steffi: Achtung für das Gesetz. Moral und Motivation bei Kant. Berlin/Boston 2013, 214-224, 274-294.

28 Kant: GMS, AA 04: 460.06f.

29 Vgl. Kant: MSTL, AA 06: 400.18-20. 


\section{Zur Begründung des Sollens}

Mein Bewusstsein des Moralgesetzes ist also der subjektive Grund dafür, dass ich verpflichtet bin. Nun bin ich aber zu nichts subjektiv verpflichtet, was nicht objektiv gültig ist. Das Moralgesetz, dessen ich mir subjektiv als verpflichtend bewusst bin, muss daher auch objektiv gültig sein.

Kant ist der Auffassung, dass sich die moralischen Gesetze als „Gesetze der Freiheit" von den Naturgesetzen darin unterscheiden, dass sie reine praktische Vernunftgesetze sind. In der Kritik der praktischen Vernunft weist er die Vernunft dementsprechend als Urheberin des Gesetzes und der Nötigung, diesem Gesetz Folge zu leisten, aus und bezeichnet das „Bewußtsein dieses Grundgesetzes [als] ein Factum der Vernunft“30. Wie Kant erklärt, lässt sich das Bewusstsein des Gesetzes weder aus anderen Vernunftbegriffen wie dem der Freiheit deduzieren noch empirisch herleiten, sondern ist „als gegeben anzusehen“"31. Eine Erklärung für unser Bewusstsein eines unbedingten Moralgesetzes kann es daher streng genommen nicht geben. Wir können lediglich erläutern: Es ist uns vernünftig gegeben. Dies bedeutet nun nicht, dass das Moralgesetz durch eine externe legislative Instanz gegeben wird. Es ist der Kern der kantischen Moralbegründung, dass das Moralgesetz ein Gesetz der Autonomie der Vernunft der den moralischen Forderungen unterworfenen Subjekte ist ${ }^{32}$ und dass diese Selbstbezüglichkeit der Vernunft die Grundlage von Verpflichtung überhaupt ausmacht. ${ }^{33}$ Das Sittengesetz ist „Factum der Vernunft“ daher nicht als ein extern erzeugtes ,factum brutum' für die Vernunft, sondern als eine ,Tat der Vernunft' im Sinne einer Handlung des dem Moralsubjekt eigenen Vernunftvermögens, deren Ergebnis das Bewusstsein des Sittengesetzes ist. ${ }^{34}$ In diesem Sinne „giebt [die Vernunft] (dem Menschen) ein allgemeines Gesetz, welches wir das Sittengesetz nennen““.35

30 Kant: KpV, AA 05: 31.24.

31 Kant: KpV, AA 05: 31.32.

32 Vgl. Kant: KpV, AA 05: 33.18f.

33 Vgl. Kant: GMS, AA 04: 439.30 - 32 und Kant: KpV, AA 05: 33.09f. Siehe dazu: Steigleder, Klaus: Kants Moralphilosophie. Die Selbstbezüglichkeit reiner praktischer Vernunft. Stuttgart, 59-115.

34 Die Unterscheidung zwischen dem ,Faktum der und dem ,Faktum für die Vernunft geht auf Beck zurück (vgl. Beck, Lewis White: A Commentary on Kant's ,Critique of Practical Reason. Chicago 1960, 168) und wird von Allison weiter ausgeführt (vgl. Allison, Henry E.: Kant's Theory of Freedom. Cambridge 1990, 230 -239). Zur Interpretation des „Factums“ als „Tat der Vernunft“ vgl. Willaschek, Marcus: Praktische Vernunft. Handlungstheorie und Moralbegründung bei Kant. Stuttgart 1992, 174-193. Wie Willaschek zeigt, handelt es sich beim „Factum“ sowohl um eine Tätigkeit, als auch um ein Ergebnis (dieser Tätigkeit); vgl. $180 \mathrm{f}$.

35 Kant: KpV, AA 05: 31.36f. 
Praktische Gesetze ,gibt‘ es folglich auch nur insofern, als sie durch Vernunft hervorgebracht werden. ${ }^{36}$

Was aber kann es heißen, dass die Vernunft ,etwas tut‘? Wie ein Blick auf die handlungstheoretischen Grundlagen von Kants Verpflichtungsbegriff zeigt, ist die das ,Sollen' erst konstituierende, Tat der Vernunft' begrifflich bereits in der Auflösung der Dritten Antinomie angelegt. Kant argumentiert dort für einen Begriff praktischer Rationalität, nach dem die Vernunft als reine Spontaneität das Vermögen der Freiheit ist. Die ,Tat der Vernunft‘ fällt dabei unter Kants allgemeinen Handlungsbegriff, demzufolge „Handlung“ alles Geschehen umfasst, das eine Wirkung hervorbringt. Dass die Vernunft etwas ,tut', heißt daher, dass sie auf eine spezifische Weise wirkt: sie hat Kausalität. Als reine Spontaneität ist die Vernunft das Vermögen der Ideen. Ihre Inhalte sind allgemein, notwendig und nicht erklärbar. Als „Fähigkeit, sich in eine andere „Ordnung der Dinge“ und damit „,in ein Verhältnis zu bestimmenden Gründen von ganz anderer Art“ zu setzen ${ }^{37}$, ist sie das Vermögen der Freiheit. Sie ist die Fähigkeit, für vernünftige Erwägungen zugänglich zu sein und sich nicht-kontingente Zwecke setzen zu können. Kant denkt somit „Sollen“ und „Freiheit“ als Wechselbegriffe: Die Vernunft ,gibt“ uns den Begriff eines unbedingt gültigen Moralprinzips. Dies bedeutet nun nichts anderes, als sich im Moment der Überlegung von den empirischen Gegebenheiten zu distanzieren und eine nicht-empirische, intelligible Perspektive einzunehmen. Die Freiheit, eine intelligible Perspektive einzunehmen, wird durch das Moralbewusstsein als „ein Factum bestätigt“. ${ }^{38}$

Kants Faktumsthese ist vor diesem Hintergrund nicht als Notbehelf, sondern als Kern seiner Moralbegründung zu verstehen. Denn sie zeigt, dass die Begründung der Moral vom verpflichteten Subjekt selbst ausgehen muss, das sich als Vernunftsubjekt selbst Zwecke vorgeben kann. Kant bringt diesen Gedanken in der Kritik der praktischen Vernunft auf den Punkt, wenn er den „Ursprung“ der Pflicht in der „Persönlichkeit“ verortet. Diese ist Ausdruck für die freie und vernünftige menschliche Veranlagung und zeigt sich insbesondere darin, „eigenthümlichen, nämlich von seiner eigenen Vernunft gegebenen, reinen praktischen Gesetzen [...] unterworfen“" zu sein. ${ }^{39}$. Moralisch verpflichtet zu sein, bedeutet demnach, dass wir uns eines moralischen Gebots bewusst sind, das seinerseits

36 Vgl. Kant: KpV, AA 05: 19.14f. Dies spricht dagegen, Kant aus metaethischer Perspektive als moralischen Realisten zu verstehen. Nach Kant gibt es keine der menschlichen Vernunft vorgeordnete moralische Realität, die durch die Vernunft eingesehen werden müsste.

37 Kant: GMS, AA 04: 457.10f.

38 Kant: KpV, AA 05: 06.11f.

39 Kant: KpV, AA 05: 86.29-87.08. 
durch die Vernunft ,konstituiert' wird. ${ }^{40}$ Wir fühlen uns subjektiv verpflichtet, da wir aufgrund unserer sinnlich-vernünftigen Doppelnatur durch das Gefühl der Achtung für ein objektives Moralprinzip empfänglich sind. Die Forderung des Moralgesetzes ist wiederum objektiv dadurch begründet, dass es ein Gesetz unserer Vernunft ist.

\section{Grenzen des kantischen Verpflichtungsbegriffs}

Verpflichtung heißt nach Kant, dass es ein Moralgesetz gibt, das wir subjektiv als bindend anerkennen und das unseren Willen objektiv bindet. Zeigen zu wollen, auf welche Weise die Vernunft als Vermögen absoluter Spontaneität das unbedingte Moralprinzips konstituiert, käme nun dem Versuch gleich, einen empirischen Beweis für die Freiheit zu liefern, die sich doch von der Naturkausalität gerade durch das nicht-empirische Merkmal ihrer Kausalität unterscheidet. Mit anderen Worten: $\mathrm{Zu}$ zeigen, wie die Vernunft handelt, hieße, einen Begriff, der selbst kein Gegenstand sinnlicher Anschauung ist, in einer solchen Anschauung darzustellen. ${ }^{41}$ Die Auseinandersetzung mit dem Begriff der Freiheit, der seinerseits eben kein „Erfahrungsbegriff“, sondern eine „Idee der Vernunft“ ist ${ }^{42}$, markiert daher für Kant nicht nur die Grenze der philosophischen Analyse von „Verpflichtung“, sondern auch die „äußerste[...] Grenze aller praktischen Philosophie“. ${ }^{43}$

Kant geht, wie in der Philosophie nicht unüblich, von bestimmten Grundannahmen aus, die ihrerseits nicht explizit begründet werden. Eine solche Grundannahme ist, dass Moral notwendig und allgemeingültig sein und daher auf apriorischen Prinzipien beruhen müsse. ${ }^{44}$ Dabei ist es die Vernunftfähigkeit, die allen Handlungssubjekten gemeinsam ist, sie zu urteilen befähigt und schließlich auf das Vernunftgesetz als moralischen Maßstab festlegt. Allgemeinheit und Notwendigkeit kennzeichnet die Gesetze der Vernunft. Daher kann nur Vernunft

40 Oliver Sensen bezeichnet Kants Position daher als „transcendental constitutivism“. Vgl. Kant’s Constructivism. In: Constructivism in Ethics. Ed. Carla Bagnoli. Cambridge 2013, 63-81, 65 f., 77. 41 „Wo aber Bestimmung nach Naturgesetzen aufhört, da hört auch alle Erklärung auf [...]“ (Kant: GMS, AA 04: 459.14f). Ebenso wenig sinnvoll wäre es, zu fragen, warum die Vernunft gerade dieses und kein anderes Moralprinzip ,gibt‘. Als Ergebnis ihrer absoluten Spontaneität ist das Moralgesetz ebenso notwendig und unerklärbar gegeben, wie die zwölf Kategorien des Verstandes. Vgl. zu dieser Parallele Sensen, Oliver: Kant's Constructivism. In: Constructivism in Ethics. Ed. Carla Bagnoli. Cambridge 2013, 63-81, 78.

42 Kant: GMS, AA 04: 455.14 und 24.

43 Kant: GMS, AA 04: 455.10, 458.37-459.02.

44 Vgl. z. B. Kant: GMS, AA 04: 389.12-23. 
Maßstabgeberin für Moral sein - vorausgesetzt, Moral muss notwendig und allgemein sein.

Wir können daher der Pflichtdefinition lediglich eine Erläuterung, jedoch keine Erklärung im Sinne eines strikten Beweises dafür hinzufügen, wie das normative Müssen entsteht. Dabei scheint es, als setze Kant die Vernunft hier als ein Axiom, das wir voraussetzen müssen, aber nicht weiter begründen können: „[D]ie vernünftige Natur existirt als Zweck an sich selbst. “45 Nun ist es, wie schon Dieter Henrich bemerkt hat, eher unwahrscheinlich, dass jemand einen positiven Beweis gegen die Vernunft und die Freiheit führen kann. ${ }^{46}$ Solange dies gilt, haben wir keinen Grund, auf Kants Grundvoraussetzung von Verpflichtung, nämlich dass wir vernünftige und daher selbstbestimmte Subjekte der Moral sind, zu verzichten. ${ }^{47}$

45 Kant: GMS, AA 04: 429.02f.

46 Henrich, Dieter: Die Deduktion des Sittengesetzes. Über die Gründe der Dunkelheit des letztes Abschnittes von Kants ,Grundlegung zur Metaphysik der Sitten'. In: Denken im Schatten des Nihilismus. Festschrift für Wilhelm Weischedel. Hg. von Alexander Schwan. Darmstadt 1975, 55-112, 112. Gegen die Möglichkeit eines Beweises der Unmöglichkeit der Freiheit vgl. Kants Auseinandersetzung mit dem Fatalisten in RezSchulz, AA 08: 13.06-14.20.

47 Für hilfreiche Anregungen zu diesem Beitrag danke ich Georg Mohr und für zahlreiche Diskussionen insbesondere zu Kants Verpflichtungsbegriff Oliver Sensen. 